\title{
Using Thinkalouds to Understand Rule Learning and Cognitive Control Mechanisms Within an Intelligent Tutoring System
}

\author{
Deniz Sonmez Unal ${ }^{1(\bowtie)}\left(\mathbb{0}\right.$, Catherine M. Arrington ${ }^{2}\left(\mathbb{0}\right.$, Erin Solovey $^{3}(\mathbb{0}$, \\ and Erin Walker ${ }^{1}$ (i) \\ 1 University of Pittsburgh, Pittsburgh, PA 15260, USA \\ \{d.sonmez, eawalker\}@pitt.edu \\ 2 Lehigh University, Bethlehem, PA 18015, USA \\ kate.arrington@lehigh.edu \\ 3 Worcester Polytechnic Institute, Worcester, MA 01609, USA \\ esolovey@wpi.edu
}

\begin{abstract}
Cognitive control and rule learning are two important mechanisms that explain how goals influence behavior and how knowledge is acquired. These mechanisms are studied heavily in cognitive science literature within highly controlled tasks to understand human cognition. Although they are closely linked to the student behaviors that are often studied within intelligent tutoring systems (ITS), their direct effects on learning have not been explored. Understanding these underlying cognitive mechanisms of beneficial and harmful student behaviors can provide deeper insight into detecting such behaviors and improve predictive models of student learning. In this paper, we present a thinkaloud study where we asked students to narrate their thought processes while solving probability problems in ASSISTments. Students are randomly assigned to one of two conditions that are designed to induce the two modes of cognitive control based on the Dual Mechanisms of Control framework. We also observe how the students go through the phases of rule learning as defined in a rule learning paradigm. We discuss the effects of these different mechanisms on learning, and how the information they provide can be used in student modeling.
\end{abstract}

Keywords: Cognitive control · Rule learning · Problem solving · Intelligent tutoring systems

\section{Introduction}

In ITS research, student behaviors that are associated with positive and negative cognitive and motivational states are often used within student models to design personalized adaptations. These states are often defined at a high level (e.g. gaming the system, zoning out), while in cognitive psychology, cognitive states are 
studied at a much finer grain. We believe that identifying the parallels between the low-level cognitive structures that are studied within controlled tasks in cognitive science literature and the student behaviors associated with both positive and negative cognitive and motivational states within ITS research will improve our understanding of student learning and eventually help us design better student models and ITSs. Two such lower-level cognitive processes are cognitive control and rule learning.

Cognitive control is the basis of goal-directed behavior. It is defined as the ability to adapt behavior depending on the current goals and online maintenance of goal-related information [3]. In cognitive psychology, cognitive control's role in self-regulating behavior [16], focusing attention [19], and goal maintenance [4] have been studied within controlled tasks. These are relevant in ITS research in detecting various kinds of student behaviors. Examples include how students inhibit their will to game the system in face of temptation [11], interfering with student zoning out [12], and supporting self-regulated learning strategies [1]. Despite the fact that low-level cognitive structures that are studied in cognitive science are the underlying mechanisms of these behaviors, these mechanisms are rarely explored directly in the ITS literature. We hypothesize that identifying these mechanisms could help us better understand student behaviors and eventually help us design better detectors of them.

Rule learning consists of activities related to collecting instances of some phenomenon and identifying commonalities, relationships, and rules from these specific instances. These activities show themselves in the learning domain in the induction and refinement processes that are introduced in [17]. Some examples for these processes are perception, generalization, discrimination, categorization, and schema induction. These processes are linked to rule learning as they also require abstracting regularities and relationships, and inducing rules from them. ITSs support induction and refinement processes by giving timely feedback, guiding students' attention, and presenting worked examples in order to achieve robust learning. However, again, the underlying cognitive mechanisms of the processes are underexplored in this line of research.

As a first step, we investigate how these low-level cognitive mechanisms can be detected within an ITS. More specifically, we are interested to discover how cognitive control and rule learning present themselves within a real setting and if they have direct effects on learning, addressing two research questions: 1) How do phases of rule learning and modes of cognitive control manifest themselves in problem solving? 2) Do different operation modes of cognitive control and the different phases of rule learning have an effect on domain learning?

We designed a thinkaloud study where we instructed students to verbalize their thoughts while solving probability problems in ASSISTments [15]. Students were randomly assigned to one of the two conditions that were designed to encourage them to use different modes of cognitive control [4], and we explored differences in student behavior and learning. In addition, we designed the problems in a way that allows us to observe the phases of rule learning within a more complex educational context. 


\section{Background}

\subsection{Cognitive Control and Dual Mechanisms Framework}

The Dual Mechanisms of Cognitive Control (DMC) framework [4] suggests that cognitive control operates via two distinct modes: proactive and reactive control. Proactive control is used when the goal-related information is actively maintained in order to prepare for cognitively demanding events. In contrast, in reactive control, goal-relevant information is only retrieved in a "just-in-time" manner and individuals rely on triggers to focus their attention back on the goal-relevant information. Even though proactive and reactive control are not considered to be mutually exclusive, people are likely to prefer one over the other. This preference is caused by individual factors such as age [20], working memory capacity [21,23], and external factors such as incentives [5], and the working memory load the task introduces [22].

To assess the relative use of proactive and reactive control, the AX continuous performance task (AX-CPT) has been used heavily $[2,4,8]$. In the AX-CPT, participants respond to letter probes based on the previous letter cue. Participants are instructed to provide a certain response if the cue-probe pair is an "AX" pair, and a different response is required for any other letter sequences. The performance of participants on specific letter sequences is indicative of their usage of proactive and reactive control. Prior research have successfully induced participants to utilize proactive or reactive control in the AX-CPT by strategy training $[5,13,14,20]$. The study we describe in this paper was inspired by this method. We test if a similar manipulation can successfully be applied to a realistic learning task. Further, we investigate if utilizing one mode of cognitive control influences learning.

\section{$2.2 \quad$ Rule Learning}

Rule learning includes investigating how humans go through phases of recognizing instances and keeping them in memory, detecting the regularities, and understanding the relationships between them [10]. The behaviors associated with the phases of rule learning were studied within different versions of a rule attainment task $[6,7,10,18]$. In one example, subjects are shown cards with sequentially-numbered circles. Exactly one of the circles is blue. The subjects must predict the position of the blue circle on the next card. In other words, they should respond in a certain way if the position of the blue circle is changing based on a rule. In all versions of the task, three main phases of rule learning were identified based on how subjects respond to the stimuli presented. These are rule search, rule discovery and rule following.

Rule Search. The first response with a new rule and all responses preceding rule discovery are identified as the rule search phase.

Rule Discovery. The third correct response in a row indicates that the subject discovered the rule. 
Rule Following. The streak of correct responses after rule discovery corresponds to rule following.

This work investigates if students learn rules associated with mathematical problems in the same way as in the rule learning paradigm. We identify possible different patterns of rule learning that can occur in a real learning environment.

\section{Task Design}

We used ASSISTments [15] to design our task. It is an online tutoring system that allows teachers to write problems with solutions, hints, and feedback. Students are assisted by the system (either on demand or automatically) with hints, scaffolding (i.e. breaking the problem down to steps), and feedback. Teachers can get immediate feedback on students' performance on the problems. We built a problem set that consisted of 9 probability problems (3 calculating probability, 3 addition rule with non-mutually exclusive events, 3 multiplication rule with dependent events). All problems were divided into 3 to 4 substeps. Participants were not expected to solve the problem when they first saw it. Instead, they were asked to rate their confidence level in solving the particular problem. When the participants clicked on the "Next Problem" ${ }^{1}$ button after confidence rating, they were shown the first substep to solve that problem in the same window (see Fig. 1). Similarly, after each substep, participants were presented with the next one until they reached the last substep that would lead them to the solution. The reasoning behind this design is to observe how the participants maintain the goal of the full problem when they needed to solve it in multiple steps.

\subsection{Cognitive Control Manipulation}

The participants were shown a prompt below the problem substep texts. The prompt instructed, "Think about how this step relates to the goal of the problem" in the proactive condition to encourage active maintenance of the goal of the full problem. In the reactive condition, it instructed, "Think about how you are solving this step" in order to make the participants only pay attention to what the substep tells them to do. The purpose of this is to induce proactive or reactive control through strategy training similar to [14]. This allowed us to observe how proactive and reactive control look like in a real problem-solving environment. The participants who were prompted to relate the substeps to the problem goal will be utilizing proactive control and the participants who were prompted to only react to the substeps will be utilizing reactive control. An example problem shown in Fig. 1.

\footnotetext{
${ }^{1}$ ASSISTments does not allow one to change the interface elements such as button text. Even though "next problem" sounds odd in this design as participants were going to "next step", we did not observe confusion among participants as they were given time to practice with this design.
} 


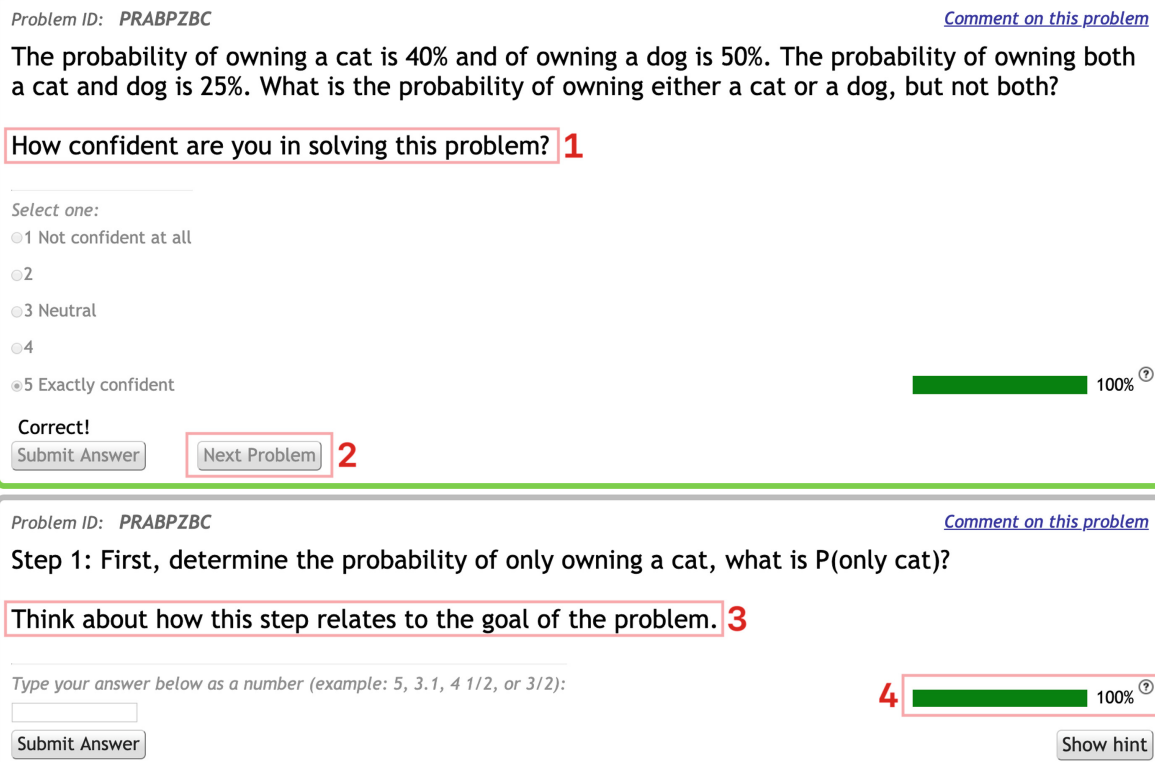

Step 1: First, determine the probability of only owning a cat, what is $\mathrm{P}$ (only cat)?

Think about how this step relates to the goal of the problem. 3

Type your answer below as a number (example: 5, 3.1, 41/2, or 3/2):

Fig. 1. 1: Participants rate their confidence level in solving the shown problem when they first see it. The system accepts all answers to confidence ratings as correct. 2: When participant clicks on "Next problem" first substep of the problem was shown. 3: Participants see a prompt below the problem text in each substep to remember the strategy they were trained to follow based on the condition they are assigned. 4: The green bar shows if student took the available hints on the step. $100 \%$ means no hints were taken. As students take more hints, the percentage decreases. (Color figure online)

\subsection{Rule Learning Manipulation}

To track how students go through the phases of rule learning, every problem substep was assigned a rule. For example, for the problem given in Fig. 1, the rules assigned to the substeps are: $P($ only $A)=P(A)-P(A \cap B)$ for substeps 1 and 2 , and $P(A$ or $B$ not both $)=P($ only $A)+P($ only $B)$ for the final substep. There were 7 distinct rules that were assigned to the problem substeps across all problems. The students may see the same rule either within a problem (as in the example) or across multiple problems. Participants saw each rule at least 3 times so that we would be able to track how they moved through phases of rule learning over multiple occurrences of each rule.

\section{Study}

The main purpose of this paper is to investigate how cognitive control and rule learning show themselves during problem solving and understand the effect of these mechanisms on learning with an ITS. Within this section we describe a thinkaloud study we designed in order to achieve these goals in more detail. 
20 undergraduate students (6 male) from the Northeastern US, between 18 and 23 years old $(M=19.45, S D=1.27)$, were recruited via emails sent to student mailing lists and flyers posted around university campuses. Our inclusion criterion was having completed no more than two university-level math courses. The study was a 1 -hour session and participants were paid $\$ 10$ compensation.

Participants solved the problems on ASSISTments version 1.0. They were provided a pen and a scratch paper to make the calculations on paper if they wished. They were also allowed to use the built-in calculator on the computer. We recorded the computer screen and thinkalouds while participants solved problems using a screen recording tool with audio.

After providing written consent, participants were introduced to ASSISTments. Participants first solved a simple practice problem to get used to the interface. We explained how they could submit answers and ask for hints as they solved the practice problem. After the practice, participants took a pre-test consisting of 6 probability problems. They solved these problems in "test mode" of ASSISTments (no hints available). After the pre-test, we gave another practice to the participants to prepare them for the thinkaloud session in which they were randomly assigned to one of the two conditions named "Proactive" and "Reactive". Within the second practice, participants solved an example problem with the prompts we described in Sect. 3.1 based on the condition they are assigned. After this practice, participants solved the real problems and engaged in the thinkaloud activity. After the thinkaloud session, participants took a post-test that was isomorphic to the pre-test, then filled a demographic questionnaire.

\section{Data Analysis}

\subsection{Data Coding}

We coded the video recordings from the thinkaloud sessions using Atlas.ti software. Each substep of the problem was coded. Our data had 532 substeps across 19 participants ( 1 participant was excluded from the analyses due to solving the pre-test in "tutor mode" of ASSISTments). The codes consisted of six labels related to the different modes of cognitive control and the phases of rule learning, and substeps could be given one or more labels. Two coders coded $20 \%$ of the data independently. Cohen's kappa was used to compare the ratings of the two coders. The agreement was $\mathrm{K}=0.70$ for the labels associated with cognitive control (relation to goal, saying answer, reacting), and $\mathrm{K}=0.82$ for the rule learning labels (rule search, rule discovery, rule following). The labels we gave the substeps are described below.

Relation to Goal. Substeps where the participant relates the current step to the goal of the problem or where they repeat the goal explicitly (e.g. "This step relates to the goal because it helps us eliminate the probability of owning both a cat and a dog.", "So, the goal of the problem is getting the probability of A or B occurring but not both."). 
Saying the Answer. Substeps where the participant says the answer but never show how the answer relates to the goal of the problem (e.g. "The answer is 35.", "Total number of possible outcomes will just be number of prizes plus number of blanks because there's a possibility of getting either of those, and that is 35.").

Reacting. Substeps where the participant reacts to a mistake or a hint (e.g. "Ok it is wrong.", "Oh, I see I did not have to do the multiplication, that was just part of it.").

Rule Search. Substeps where the participant is simply guessing the answer or trying to figure out the right way to solve it (e.g. "I don't quite remember how to solve this but I'm going to try multiplying them before I take the hint.", "So, my first inclination is to do something with 0.3 and 0.4 . I am going to try multiplying them or maybe I should add them.").

Rule Discovery. Substeps where the participant has just discovered a rule ("Oh! So, we add the probability of just A and just B.").

Rule Following. Substeps where the participant explains how they got to an answer. Participants being in this state does not mean they follow the correct rule. Sometimes they follow a rule they think is correct (e.g. "We multiply the two probabilities together and that is 5/44.").

\subsection{Statistical Analyses}

In order to test if the students in the experimental conditions are behaving as expected, we conducted two-sample t-tests for each code. The results suggested that the participants in the proactive condition related the problem substeps to the goal significantly more than the ones in the reactive condition $(t(17)=2.49, p<0.05)$. In contrast, participants in the reactive condition answered by simply saying the answer significantly more than the participants in the proactive condition $(t(17)=-2.66, p<0.05)$. There was no significant difference in reacting to hints or mistakes between two conditions $(t(17)=-0.55, p=0.59)$. Table 1 summarizes these results. Overall, the results suggest that our experimental conditions were successful.

Table 1. Mean (SD) of quotation labels between the experimental conditions. * indicates $p<0.05$.

\begin{tabular}{l|l|l|l}
\hline Condition & Relation to goal & Saying the answer & Reacting \\
\hline Proactive & $\mathbf{7 . 8 9}(\mathbf{9 . 9 3})^{*}$ & $17.44(9.26)$ & $3.33(2.12)$ \\
\hline Reactive & $0.1(0.31)$ & $\mathbf{2 5 . 4}(\mathbf{1 . 9 6})^{*}$ & $3.8(1.55)$ \\
\hline
\end{tabular}

We could successfully alter the student behavior in a way that reflects proactive and reactive modes of cognitive control. But does using one of these modes while problem solving result in better learning gains? We conducted a 
two-way repeated-measures ANOVA to compare the pre and post-test scores between conditions. Results showed that there was no significant difference in learning gain (difference between post-test and pre-test) between the proactive $(M=0.48, S D=0.18)$ and the reactive $(M=0.32, S D=0.23)$ conditions $(F(1,17)=0.17, p=0.68)$. Note that there was no significant difference in students' pre-test scores between the proactive $(M=0.42, S D=0.24)$ and the reactive $(M=0.48, S D=0.18)$ conditions $(t(16.44)=-0.69, p=0.5)$. However, time spent on problem substeps was significantly higher in the proactive condition $(M=21.43, S D=12.89)$ than in the reactive condition $(M=17.06, S D=$ $11.19),(t(661.49)=4.77, p<0.001)$.

Next, we turn to rule learning. In our problem set, each problem substep was assigned one rule and the students may go through multiple phases of rule learning solving one substep. We identified which rule learning phases the students went through in one problem step based on the presence of the relevant labels from thinkaloud data. Then we investigated patterns of rule learning phases that the students follow across all substeps that are assigned the same rule. We extracted one sequence of rule learning phases for each participant and rule type. The unique sequences of these phases pointed out 3 different patterns (see Fig. 2 for examples) the students followed:

1) "Search, Discover, Follow" Students search for the rule that is assigned to the problem step and discover the rule either by thinking it through or asking for a hint in the first occurrences of a problem step that is assigned the particular rule. In the next occurrences, students would discover then follow that rule. This is the pattern we would see in the rule learning task.

2) "Follow_wrong, Search, Discover, Follow" Students who follow this pattern start from following a wrong rule that they think is correct. When they realize it is wrong, they search for the correct one. In the next occurrences, students would discover then follow that rule. The difference between this and the first pattern is that students already have an idea about what the rule is from the beginning. This pattern is also different than what we would see in a rule learning task. Since the rules are random in a rule learning task, the participants can only guess the rule.

3) "Follow" The student knows the rule assigned to the problem step already, and they continue following that rule in all occurrences of the same rule.

We identified different combinations of rule learning phases over multiple occurrences of the same rule type across all participants. Since we see no interaction between the experimental conditions and following specific patterns of rule learning, we investigate how students follow these patterns across conditions.

To see if these patterns were related to students' prior knowledge and learning gains, we first divided the students into 2 groups using a mean split on the learning gain. Similarly, both groups were divided into two using a mean split on the pre-test scores. In the end, we had 4 groups of students: high knowledge and high learning gain $(\mathrm{HH})(N=6)$, high knowledge and low learning gain $(\mathrm{HL})$ $(N=4)$, low knowledge and high learning gain $(\mathrm{LH})(N=3)$, and low knowledge and low learning gain (LL) $(N=6)$. Figure 3 visualizes the proportions of the 

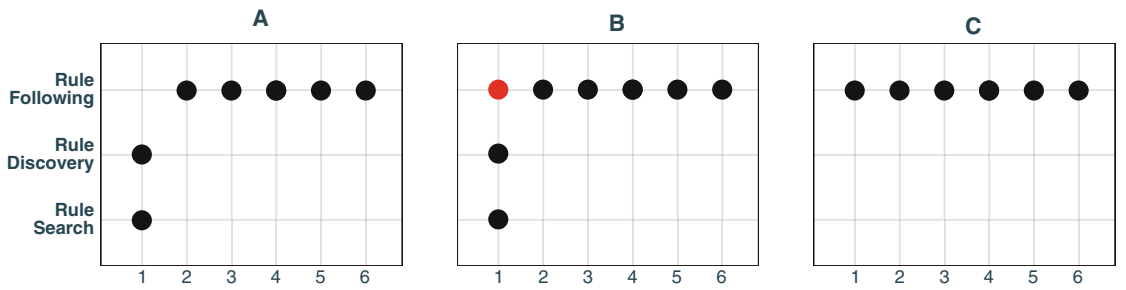

Occurrence of rule

Fig. 2. A: "Search, Discover, Follow" pattern, B: "Follow_wrong, Search, Discover, Follow" pattern (red dot represents that the participant started by following the wrong rule), C: "Follow" pattern. (Color figure online)

patterns we defined earlier for each student profile. The proportion of the rule learning patterns seem similar for different student groups except students in LH seem to follow "Search, Discovery, Follow" pattern more frequently.

To explore the difference in proportion of "Search, Discover, Follow" pattern among the different student profiles, we computed confidence intervals for these proportions. For the HL, HH, LL, and LH groups, the confidence intervals were $90 \%$ CIs $[.02, .24],[.04, .20],[.09, .32]$, and $[.31, .69]$, respectively. These intervals suggest the proportion of students who follow "Search, Discover, Follow" pattern will be higher in LH group than it is in other groups. However, this should be confirmed with a significance test with enough sample size.

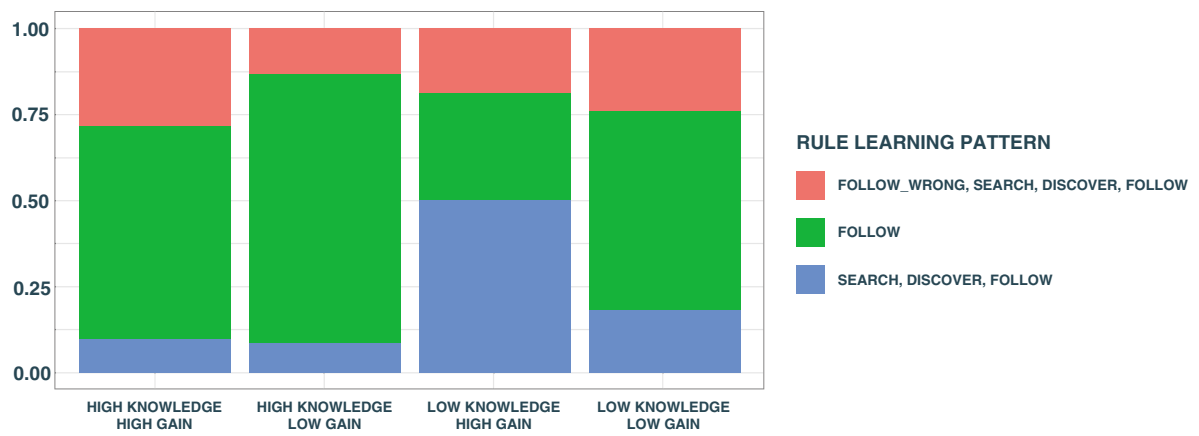

Fig. 3. Rule learning patterns by student profiles based on students' learning gains and prior knowledge.

\section{Discussion and Conclusion}

Most well-studied cognitive and motivational states that were associated with learning within ITSs have roots in cognitive science studies of rule learning and 
cognitive control. To explore the relevance of these lower level cognitive mechanisms within ITS research, we investigated how these mechanisms show themselves in a complex learning environment and their relationship with learning. We presented a study in which we induced one of the two modes of cognitive control based on the DMC framework [4] and observed how students move through the phases of rule learning within an ITS.

Our results indicated success in shifting student behavior in a way that reflects the proactive or reactive modes of cognitive control, achieving effects similar to studies that have been done with more controlled tasks $[5,13,14,20]$. However, we did not observe a difference in learning between the two modes. Multiple explanations could account for this result. Firstly, since this was a thinkaloud study, all participants were explaining their thought processes while they were solving the problems. The explanation practice might have helped all participants [9] and possibly hindered the effect of relating to the goal of the problem. Future studies without the thinkaloud procedure should further explore these effects. Secondly, since using proactive control is more cognitively demanding than reactive control [22], practicing proactive control might have exhausted the cognitive resources participants have and as a result they struggled with the learning tasks, cancelling out the benefits of maintaining the goal in working memory. Our finding on time data was consistent with this explanation as students in the proactive condition spent more time on the problem steps. As we show it is possible to induce proactive control, we hope future work will further explore its effects on learning.

Our analyses on rule learning revealed that students were following three main patterns of rule learning phases while they are solving problems. Results showed that the students with low prior knowledge and high learning gains followed the pattern that formal studies of rule learning $[6,7,10,18]$ show (i.e. "Search, Discover, Follow") more frequently than the other student profiles. This result could be an indicator of a relationship between rule learning and domain learning and ITSs can benefit from this relationship in task selection by choosing tasks that support appropriate rule learning patterns based on the student's profile. However, the small sample size was a limitation for further exploration of this relationship.

To summarize our contributions, we presented a novel coding scheme in order to categorize student utterances that are indicative of mechanisms of cognitive control and rule learning within a complex learning environment and we took a first step towards understanding the underlying mechanisms of student cognitive states that are associated with learning. We believe that identifying these underlying mechanisms within such complex learning environments will open new paths in ITS research and student modeling.

Acknowledgements. This work was supported by the National Science Foundation Award No. DGE-1835251. We would like to thank the ASSISTments team for their assistance and support, and Zachary Pixley for his help with data coding. 


\section{References}

1. Aleven, V.A., Koedinger, K.R.: An effective metacognitive strategy: learning by doing and explaining with a computer-based cognitive tutor. Cogn. Sci. 26(2), 147-179 (2002)

2. Barch, D.M., et al.: CNTRICS final task selection: working memory. Schizophr. Bull. 35(1), 136-152 (2009)

3. Botvinick, M.M., Braver, T.S., Barch, D.M., Carter, C.S., Cohen, J.D.: Conflict monitoring and cognitive control. Psychol. Rev. 108(3), 624 (2001)

4. Braver, T.S.: The variable nature of cognitive control: a dual mechanisms framework. Trends Cogn. Sci. 16(2), 106-113 (2012)

5. Braver, T.S., Paxton, J.L., Locke, H.S., Barch, D.M.: Flexible neural mechanisms of cognitive control within human prefrontal cortex. Proc. Natl. Acad. Sci. 106(18), 7351-7356 (2009)

6. Burgess, P.W., Shallice, T.: Bizarre responses, rule detection and frontal lobe lesions. Cortex 32(2), 241-259 (1996)

7. Cao, B., Li, W., Li, F., Li, H.: Dissociable roles of medial and lateral PFC in rule learning. Brain Behav. 6(11), e00551 (2016)

8. Chatham, C.H., Frank, M.J., Munakata, Y.: Pupillometric and behavioral markers of a developmental shift in the temporal dynamics of cognitive control. Proc. Natl. Acad. Sci. 106(14), 5529-5533 (2009)

9. Chi, M.: Self-explaining expository texts: the dual processes of generating inferences and repairing mental models. Adv. Instr. Psychol. 5, 161-238 (2000)

10. Crescentini, C., Seyed-Allaei, S., De Pisapia, N., Jovicich, J., Amati, D., Shallice, T.: Mechanisms of rule acquisition and rule following in inductive reasoning. J. Neurosci. 31(21), 7763-7774 (2011)

11. Dang, S., Koedinger, K.: Exploring the link between motivations and gaming. In: EDM (2019)

12. D'Mello, S., Olney, A., Williams, C., Hays, P.: Gaze tutor: a gaze-reactive intelligent tutoring system. Int. J. Hum. Comput. Stud. 70(5), 377-398 (2012)

13. Edwards, B.G., Barch, D.M., Braver, T.S.: Improving prefrontal cortex function in schizophrenia through focused training of cognitive control. Front. Hum. Neurosci. 4, $32(2010)$

14. Gonthier, C., Macnamara, B.N., Chow, M., Conway, A.R., Braver, T.S.: Inducing proactive control shifts in the AX-CPT. Front. Psychol. 7, 1822 (2016)

15. Heffernan, N.T., Heffernan, C.L.: The assistments ecosystem: building a platform that brings scientists and teachers together for minimally invasive research on human learning and teaching. Int. J. Artif. Intell. Educ. 24(4), 470-497 (2014)

16. Koch, S., Holland, R.W., van Knippenberg, A.: Regulating cognitive control through approach-avoidance motor actions. Cognition 109(1), 133-142 (2008)

17. Koedinger, K.R., Corbett, A.T., Perfetti, C.: The knowledge-learning-instruction framework: bridging the science-practice chasm to enhance robust student learning. Cogn. Sci. 36(5), 757-798 (2012)

18. Li, F., Cao, B., Gao, H., Kuang, L., Li, H.: Different brain potentials evoked at distinct phases of rule learning. Psychophysiology 49(9), 1266-1276 (2012)

19. Mackie, M.A., Van Dam, N.T., Fan, J.: Cognitive control and attentional functions. Brain Cogn. 82(3), 301-312 (2013)

20. Paxton, J.L., Barch, D.M., Storandt, M., Braver, T.S.: Effects of environmental support and strategy training on older adults' use of context. Psychol. Aging 21(3), 499 (2006) 
21. Redick, T.S.: Cognitive control in context: working memory capacity and proactive control. Acta Psychol. 145, 1-9 (2014)

22. Speer, N.K., Jacoby, L.L., Braver, T.S.: Strategy-dependent changes in memory: effects on behavior and brain activity. Cogn. Affect. Behav. Neurosci. 3(3), 155-167 (2003)

23. Wiemers, E.A., Redick, T.S.: Working memory capacity and intra-individual variability of proactive control. Acta Psychol. 182, 21-31 (2018) 